\title{
Hemolytic Disease of the Newborn Due to Anti-Rhesus (E) Alloimmunization
}

\author{
Divya Rai ${ }^{1}$ and Manish Raturi ${ }^{2 *}$ \\ ${ }^{\prime}$ Department of Pathology, Fatima Hospital, Mother Teresa Road, Padri Bazar PO. Gorakhpur, Uttar Pradesh, India \\ ${ }^{2}$ Department of Immunohematology and Blood Transfusion, Himalayan Institute of Medical Sciences, Swami Rama Himalayan University, Swami Ram Nagar, Jolly \\ Grant, Dehradun, Uttarakhand, India
}

\begin{abstract}
There is a paucity of data on the prevalence of the Rhesus (Rh) induced erythrocyte alloimmunization other than the notorious Rh-D antigen among the Indians. This is evident more so mainly due to the sub-optimal antenatal care provided to the women here. We describe herein, a case of hemolytic disease of the newborn due to anti-Rh (E) specificity in a newborn of an O Rh (D) positive first-time mother, who hailed from a rural background in India. The baby's rising bilirubin levels [ $>20$ $\mathrm{mg} / \mathrm{dL}$ ] warranted an urgent double volume exchange transfusion. Gradually the baby's clinical condition improved and was discharged from the hospital on the 19th day of his birth. Currently, the baby is doing well with optimal developmental milestones.
\end{abstract}

Keywords: Hemolytic disease of the newborn, Maternal immunization, Red cell alloimmunization, Pregnancy complications

\section{Background}

The frequency of the hemolytic disease of the newborn (HDN) with indirect hyperbilirubinemia due to Rh (D) sensitization has reduced due to the widespread use of anti-D intravenous $\gamma$-globulin (IVIG) these days [1]. Hence, there is a proportional increase in the world-wide recognition of the importance of the minor blood group incompatibility such as Kell and the Rhesus (Rh) non-D erythrocyte alloimmunization as the cause of HDN [2]. Till date, $49 \mathrm{Rh}$ antigens are known. Antigens D, C, E, c, and e are among the most significant antigens. Disease intensity for the erythrocyte alloimmunization depends on the type of antigen, degree of hemolysis and the immune response of the mother [whether, she is a responder or not]. There is, nevertheless, still a paucity of data on the prevalence of Rh induced erythrocyte alloimmunization other than the notorious $\mathrm{Rh}-\mathrm{D}$ antigen among the Indian population. This is evident more so mainly due to the sub-optimal ante-natal care available to the pregnant women in our nation. Herein, we report a case of HDN due to Rh non-D i.e. anti-Rh (E) alloimmunization in the mother and its timely management to help improve her baby's optimal clinical outcome.

\section{Case Report}

A term 37-week gestation, male baby with a birth weight of 1,800 g [small for gestational age (SGA)] was born to a 27-year-old G1P1 Indian mother, by an uneventful vaginal delivery at a 200 bedded tertiary care academic hospital in the Gorakhpur district of eastern Uttar Pradesh, India. On detailed history, she mentioned that she belonged to a rural background and had visited the primary health centre [PHC] situated in her village just once, in her first trimester. She received no antenatal care including fetal wellbeing monitoring during the course of her pregnancy. Her only medication history was iron and folate supplements. Importantly, she received one unit of whole blood ( $450 \mathrm{ml})$ transfusion at the same visit due to the marked anemia. She was referred to our hospital at the $37^{\text {th }}$ week of gestation due to her labor pains.

On physical examination, this was an SGA baby with pallor and deep icterus till the soles. The newborn was active, alert with good spontaneous movements and normal neonatal reflexes. Although, his respiratory and cardiovascular findings were within normal limits, he had moderate hepatomegaly on abdominal examination [1.0 cm below the right costal margin]. Risk factors for neonatal sepsis, and gross congenital anomalies were absent. His total serum bilirubin was $461.7 \mu \mathrm{mol} / \mathrm{L}$ at $18 \mathrm{~h}$ after birth (in newborns, the total serum bilirubin would normally range from 34.0 to $110 \mu \mathrm{mol} / \mathrm{L}$ within $24 \mathrm{~h}$ of birth). He was transferred to neonatal intensive care unit [NICU] for an early management of his rising bilirubin concentration.

*Correspondence to: Dr. Manish Raturi, Department of Immunohematology and Blood Transfusion, Himalayan Institute of Medical Sciences, Swami Rama Himalayan University, Swami Ram Nagar, Jolly Grant, Dehradun (Uttarakhand) - 248001, India, E-mail: manishraturi@srhu.edu.in, ORCID ID: https://orcid.org/0000-0001-7880-7988

Citation: Rai D, Raturi M (2020) Hemolytic Disease of the Newborn Due to Anti-Rhesus (E) Alloimmunization. J Clin Anat Pathol, 5(1): 101. DOI: https://doi. org/10.47275/2332-4864-101.

Received: April 24, 2020; Accepted: May 15, 2020; Published: May 17, 2020

Copyright: (c) 2020 Rai D, et al. This is an Open Access article distributed under the terms of the Creative Commons Attribution 4.0 International License (CCBY) (http://creativecommons.org/licenses/by/4.0/) which permits commercial use, including reproduction, adaptation, and distribution of the article provided the original author and source are credited. 


\section{Immunohematology Laboratory Workup}

Mother's blood group by conventional tube technique [CTT] was O Rh D positive and was Rh-subtyped as $R_{0} R_{0}(D c e / D c e)$. As a part of her pre-transfusion work-up in accordance to the existing national guidelines [3], an antibody screen was done using commercial ID-Diacell I-II-III antibody screening panel (Biorad, Switzerland) and ID-cards "LISS/Coombs". Her antibody screening test was positive. To confirm the specificity, an 11-cell antibody identification panel (Biorad Switzerland) was utilized. The antibody was eventually identified as anti-Rh (E) which showed dosage effect. The newborn's blood group by the CTT method was B Rh D positive. In view of a positive direct coombs test [grade 3+], the baby's $\mathrm{Rh}$ sub-typing could not be performed.A possibility of the ABO HDN due to anti-B antibodies was considered. However, the eluate prepared [by heat elution method] from the baby's red cells showed anti-E specificity against both $\mathrm{O}(\mathrm{B}-\mathrm{E}+$ ) and B+E+ typed erythrocytes (Table 1). A final diagnosis of neonatal hyperbilirubinemia in a term SGA newborn due to minor incompatibility [anti-Rh (E)] was made. The neonate became more anemic on the $3^{\text {rd }}$ day of life, with a hemoglobin level of $8.2 \mathrm{~g} / \mathrm{dL}$. The peripheral blood smear showed numerous spherocytes with moderate anisopoikiolocytosis consistent with an ongoing in-vivo hemolysis (Figure 1).

Table 1: Reaction patterns of the eluate prepared from the newborn's erythrocytes.

\begin{tabular}{|l|l|l|l|}
\hline S. No & Antigen Positive Red Cells & Reaction Pattern (Agglutination grade* & Final Interpretation \\
\hline 1 & B + E + & Positive [3+] & Could be either anti-B, anti-E or both \\
\hline 2 & B + E- & Negative [0] & Anti-B ruled out \\
\hline 3 & O+E+ & Positive [3+] & Anti-E specificity confirmed \\
\hline 4 & O+E- & Negative [0] & Anti-B ruled out + Anti-E specificity confirmed \\
\hline
\end{tabular}

*Agglutination grade is read from 0 , which is a negative result, to $4+$, which is the strongest possible agglutination.

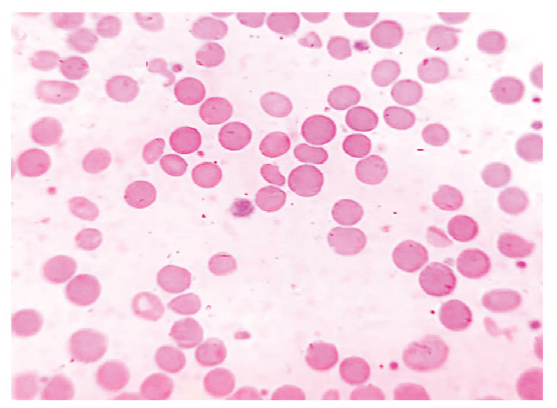

Figure 1: Peripheral blood film of the newborn showing abundant spherocytes.

\section{Biochemical Investigations}

Liver enzymes such as alkaline phosphatase, alanine transaminase, and aspartate amino transferase were all within the normal ranges. His G6PD screen was negative. His total serum bilirubin was raised from $105.3 \mu \mathrm{mol} / \mathrm{L}$ at $3 \mathrm{~h}$ of birth to as high as $461.7 \mu \mathrm{mol} / \mathrm{L}$ at $18 \mathrm{~h}$ after birth. His serum LDH levels were also considerably high (790 IU/L).

\section{Selection of Blood and Double Volume Exchange Transfusion [DVET]}

Such a critical rise of his total serum bilirubin concentration within $24 \mathrm{~h}$ of birth warranted a justifiable decision of performing a DVET. For this, the component selection, compatibility testing and administration of reconstituted whole blood [RWD] were done in accordance to the national guidelines [3]. We took an O Rh D positive, E antigen negative, leukocyte-reduced packed red blood cell [PRBC] in CPDA-1 anticoagulantpreservative solution, stored up to 5 days after collection, and reconstituted it with the thawed AB plasma to reduce the hematocrit to 50 to $60 \%$. The selected PRBC unit was cross-matched with the mother's serum using the column agglutination gel technique on commercial DiaMed LISS/ Coombs cards (Biorad, Switzerland) and was found compatible. A total volume of $288 \mathrm{ml}$ RWD was used for DVET. Additionally, the patient was managed with phototherapy. On $3^{\text {rd }}$ day post DVET, a top-up transfusion of $30 \mathrm{~mL}$ AHG cross-matched compatible, leukoreduced and E antigen negative, PRBC was also given to the newborn.

\section{Clinical Condition}

His jaundice reduced gradually. At the time of discharge his total serum bilirubin level was $161 \mu \mathrm{mol} / \mathrm{L}$ on the $19^{\text {th }}$ day of birth. The newborn showed optimal developmental milestones, consistent improvement in his body weight and a normal liver size on his follow-up visit.

\section{Discussion}

The Rh blood group system is one of the most polymorphic and immunogenic systems known in humans [4]. They are frequently associated with HDN, when fetal red blood cells (RBCs), with an antigen that the mother lacks, cross the placenta into the maternal circulation, where they stimulate immune response followed by anti-Rh antibody production. The primary maternal immune response on exposure to fetus is the production of IgM isotype. The more profound maternal IgG response is seen in subsequent pregnancies [5]. These IgG antibodies are against the fetal red cells, and can pass through the placenta and destroy the antigen-positive fetal red cells [6]. Our case is unusual in the fact that it is a unique example of moderate level anti-Rh (E) alloimmunization that happened in first pregnancy itself as opposed to the classic hemolytic severity that 
usually presents in subsequent pregnancies. This could most probably be explained by the fact that she had received a whole blood transfusion with a unit of unknown E antigen status in her first trimester at a PHC in her village. The D antigen accounts for 50\% of erythrocyte alloimmunization among ante-natal mothers [2]. However, sensitization to Rh antigens other than D i.e. The CDE system is not uncommon and these antibodies may lead to severe hemolysis in the newborns as well [7].

Mild to severe cases of HDN have been reported when anti-c, C, E or Duffy, were found in the sera of mothers. Babinszki et al have shown that the combination of anti-c and anti-E antigens can cause the occurrence of severe hemolysis in neonates [8]. This is due to the fact that most white people have R1R1 phenotype [CDe/CDe]. Our case is an unusual one, since the lady was typed as O Rh D positive and was further Rhsubtyped as $R_{0} R_{0}(\mathrm{Dce} / \mathrm{Dce})$, which has an uncommon prevalence among the Rh positive population in India. In a study conducted by Makroo et al., they reported the prevalence of the Dce/Dce phenotype as 1.15\%, that ranged from $1.06 \%$ to $1.24 \%$ in North India [9].

Furthermore, the literature shows that there is a gradual rise in the reporting of various non-Rh erythrocyte alloantibodies leading to HDN. The most well-known non-RH antigens causing neonatal HDN include Kell, Duffy, Diego, Kidd and MNS antigens causing 3\% of HDN [10]. His peripheral smear showed an abundance of spherocytes along with coombs positive hyperbilirubinemia, pointing towards an immunological cause of the on-going in-vivo hemolysis in the newborn. In most parts of our country, the quality of antenatal care (ANC) observed continues to be suboptimal. The disadvantaged women from north India are often denied the optimum quality of the ANC, compared with the southern part of India where the prenatal care is far more available to women [11]. Several factors, including reduced numbers of employment, low household income, and lack of education among their husbands directly influence the quality of the ANC and its primary utilization among women in our country. The management of anti-E alloimmunization or alloimmunization with any other erythrocyte alloantibody is similar to the management of anti-Rh (D) immunized pregnancy with a specification that blood used for either DVET or neonatal top-up transfusion should be negative for the specific antigen. Further, IVIG has been shown to reduce the need for exchange transfusions in both Rh and ABO HDN. Although data is limited, it is quite reasonable to assume that IVIG may be helpful in the non-D Rh HDN such as anti-C and anti-E [12]. The IVIG however, could not be given in our case due to cost constraints.

\section{Conclusion}

We conclude that routine erythrocyte antibody screenings in the antenatal period in terms of rhesus subgroup incompatibilities are critical for better prediction and early diagnosis of both Rh (D) as well as Rh-non(D) HDN. Optimal quality of the antenatal care is an immediate need of the hour.

\section{Conflicts of Interest:}

The authors certify that they do not have any conflict of interests.

\section{Financial Support}

Nil.

\section{References}

1. Agrawal A, Hussain KS, Kumar A. 2020. Minor blood group incompatibility due to blood groups other than Rh(D) leading to hemolytic disease of fetus and newborn: a need for routine antibody screening during pregnancy. Intractable Rare Dis Res 9(1): 43-47.https://doi.org/10.5582/irdr.2019.01094

2. Dean L. 2006. The Rh blood group. In: Blood Groups and Red Cell Antigens. National Center for Biotechnology Information, Bethesda.

3. Saran RK. 2003. Hemolytic Disease of the New Born (HDN). In: Transfusion medicine technical manual DGHS. Ministry of Health and Family Welfare, pp: 175-192.

4. Avent ND, Reid ME. 2000. The Rh blood group system: a review. Blood 95(2): 375-387.PMID: 10627438

5. Nassar GN, Wehbe C. 2020. ErythroblastosisFetalis. [Updated 2019 Nov 24]. In: Stat Pearls [Internet]. Treasure Island (FL): Stat Pearls Publishing.PMID: 30020664

6. Usman AS, Mustaffa R, Ramli N, Diggi SA. 2013. Hemolytic disease of the fetus and newborn caused by anti-E. Asian J Transfus Sci 7(1): 84-85.https://doi.org/10.4103/09736247.106750

7. Abourazzak S, Hajjaj S, Hakima C, Bouharrou A, Hida M. 2009. Haemolytic disease of the newborn due to anti-c. BMJ Case Rep 2009: bcr09.2008.0987.https://doi.org/10.1136/ bcr.09.2008.0987

8. Babinszki A, Berkowitz RL. 1999. Haemolytic disease of the newborn caused by anti-c, anti-E and anti-Fya antibodies: report of five cases. Prenat Diagn 19(6): 533-536.https://doi. org/10.1002/(sici)1097-0223(199906)19:6<533::aid-pd570>3.0.co;2-5

9. Makroo R, Gupta R, Bhatia A, Rosamma NL. 2014. Rh phenotype, allele and haplotype frequencies among 51,857 blood donors in North India. Blood Transfus 12(1): 36-39.https:// doi.org/10.2450/2013.0300-12

10. Lenkiewicz B, Zupanska B. 2003. Significance of alloantibodies other than anti-D hemolytic disease of the fetus and newborn (HDF/N).Ginekol Pol74(1): 48-54.PMID: 12715437

11. Rani M, Bonu S, Harvey S. 2008. Differentials in the quality of antenatal care in India. Int J Qual Health Care 20(1):62-71.https://doi.org/10.1093/intqhe/mzm052

12. Subcommittee on Hyperbilirubinemia. 2004. Management of hyperbilirubinemia in the newborn infant 35 or more weeks of gestation. Pediatrics 114: 297-316.https://doi.org/10.1542/ peds.114.1.297 